\title{
Effect of particle size on the formation of adiabatic shear band in particle reinforced metal matrix composites
}

\author{
L.H. Dai*, L.F. Liu, Y.L. Bai \\ State Key Laboratory of Nonlinear Mechanics (LNM), Institute of Mechanics, Chinese Academy of Sciences, Beijing 100080, PR China
}

Received 15 July 2003; received in revised form 24 October 2003; accepted 29 October 2003

\begin{abstract}
In this paper, the effect of particle size on the formation of adiabatic shear band in $2024 \mathrm{Al}$ matrix composites reinforced with $15 \%$ volume fraction of 3.5, 10 and $20 \mu \mathrm{m} \mathrm{SiC} \mathrm{particles} \mathrm{was} \mathrm{investigated} \mathrm{by} \mathrm{making} \mathrm{use} \mathrm{of} \mathrm{split} \mathrm{Hopkinson} \mathrm{pressure} \mathrm{bar} \mathrm{(SHPB).} \mathrm{The} \mathrm{results} \mathrm{have}$ demonstrated that the onset of adiabatic shear banding in the composites strongly depends on the particle size and adiabatic shear banding is more readily observed in the composite reinforced with small particles than that in the composite with large particles. This size dependency phenomenon can be characterized by the strain gradient effect. Instability analysis reveals that high strain gradient is a strong driving force for the formation of adiabatic shear banding in particle reinforced metal matrix composites (MMCp).
\end{abstract}

(C) 2004 Elsevier B.V. All rights reserved.

Keywords: Composite materials; Deformation and fracture; Shear band; Size effect; Strain gradient

\section{Introduction}

Adiabatic shear banding is a commonly observed phenomenon in various classes of materials deformed at high strain rates. Usually, the formation of adiabatic shear band is followed by macroscopic failure of materials. Therefore, an in-depth understanding in adiabatic shear banding is of great practical interest. During the past several decades, extensive research activities have been made to explore the formation and evolution of shear bands $[1-10]$. The primary mechanism for shear bands is the thermomechanical mechanism. Due to interaction of microstructures of materials, the initial homogeneous pattern of plastic deformation may be broken down into inhomogeneous one. At high strain rate of loading, this inhomogeneous deformation will induce local intensive heating which in turn leads to material softening so that the localized deformation bands are finally formed.

However, the great majority of the aforementioned efforts were focused on monolithic materials. Experimental works have demonstrated that adiabatic shear banding not only can be found in monolithic materials, but also can be observed in

\footnotetext{
* Corresponding author. Tel.: +86-10-62616852; fax: +86-1062561284

E-mail address: 1hdai@1nm.imech.ac.cn (L.H. Dai).
}

particle reinforced metal matrix composites (MMCp) $[11,12]$. Up to now, relatively limited investigations have been made to address the mechanisms for the formation and development of adiabatic shear banding in MMCp. Therefore, how to understand the effects of particle size on adiabatic shear banding in two-phase MMCp is still open.

In view of the aforementioned observations, the effect of reinforcing particle size on the formation of adiabatic shear band in $\mathrm{SiCp} / 2024 \mathrm{Al}$ matrix composites was investigated in this paper by making use of split Hopkinson pressure bar (SHPB). The mechanism for the size-dependent adiabatic shear banding phenomenon in MMCp was elucidated in this paper.

\section{Experimental procedure}

\subsection{Materials and specimens}

In the present work, MMCp have been manufactured following a PM route: mixing, compacting, and hot extrusion. The aluminum alloy 2024 was chosen as the matrix, and $15 \%$ volume fraction of $3.5,10$ and $20 \mu \mathrm{m} \mathrm{SiC}$ particles were used as the reinforcements. For dynamic compression tests, specimens were machined to short cylindrical bars with $5 \mathrm{~mm}$ in diameter and length. The extrusive direction is 
parallel to the compression axis. Before testing, samples were solution heat-tested, heated at $530{ }^{\circ} \mathrm{C}$ for $55 \mathrm{~min}$, then water-quenched.

\subsection{Dynamic compression testing}

To study the effect of particle size on the formation of adiabatic shear banding in $\mathrm{SiCp} / 2024 \mathrm{Al}$ matrix composites, a series of dynamic compression tests at high strain rates $\left(\sim 2000 \mathrm{~s}^{-1}\right)$ were performed with a split Hopkinson pressure bar (SHPB). According to the incident, reflected, and transmitted pulses which were measured by two sets of strain gauges mounted on the input and output bars, respectively, the average stress and strain in the specimen can be calculated. Details of testing can be found elsewhere $[13,14]$. After testing, the loaded specimens were sectioned along the compression axis, polished and etched for microscopic examination.

\section{Experimental results}

\subsection{Stress-strain curves}

Typical stress-strain curves of materials are shown in Fig 1. One of the prominent features in these curves is that, under the same loading condition, the flow stress of the composites depends on the SiCp particle size: the smaller the SiCp particles, the higher the flow stress of composites. Among them, the composite containing 3.5 $\mu \mathrm{m} \mathrm{SiC} \mathrm{particles} \mathrm{is} \mathrm{of} \mathrm{the} \mathrm{highest} \mathrm{flow} \mathrm{stress} \mathrm{and} \mathrm{the}$ matrix material, $2024 \mathrm{Al}$ alloy, does the lowest flow stress under the same loading condition. We have demonstrated in our previous works $[15,16]$ that this size-dependent strengthening behavior of MMCp can be characterized

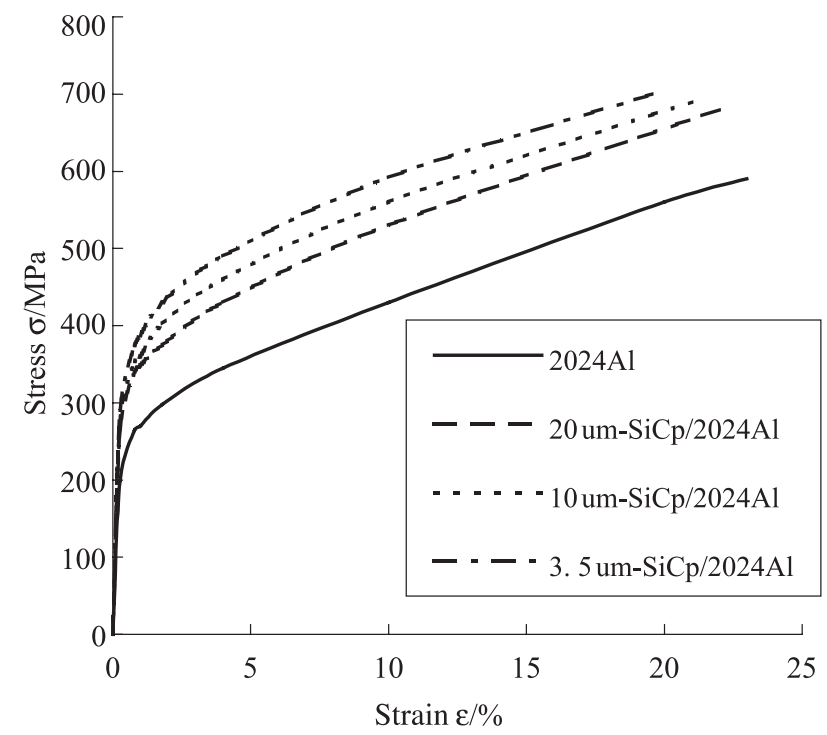

Fig 1. Dynamic compressive stress strain curves of $\mathrm{SiCp} / 2024 \mathrm{Al}$ composites : (a) $3.5-\mu \mathrm{m}$ particle; (b) $10-\mu \mathrm{m}$ particle; c) $20-\mu \mathrm{m}$ particle. (a)

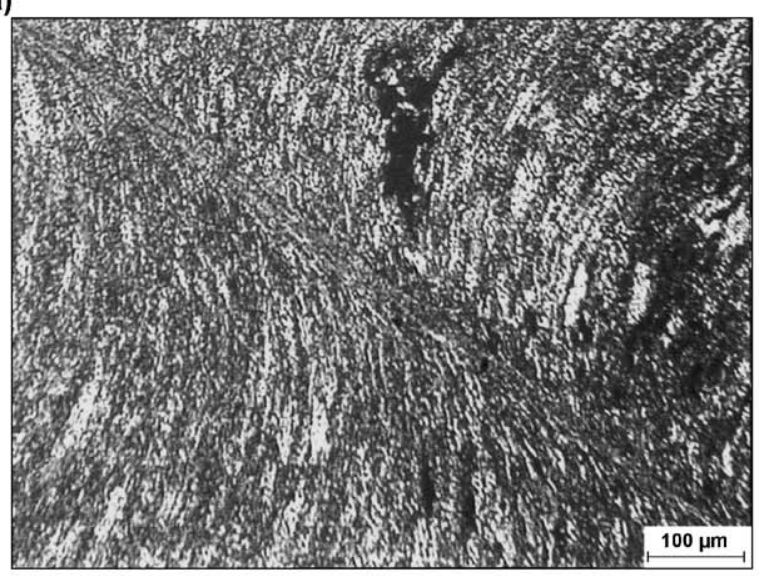

(b)

(c)
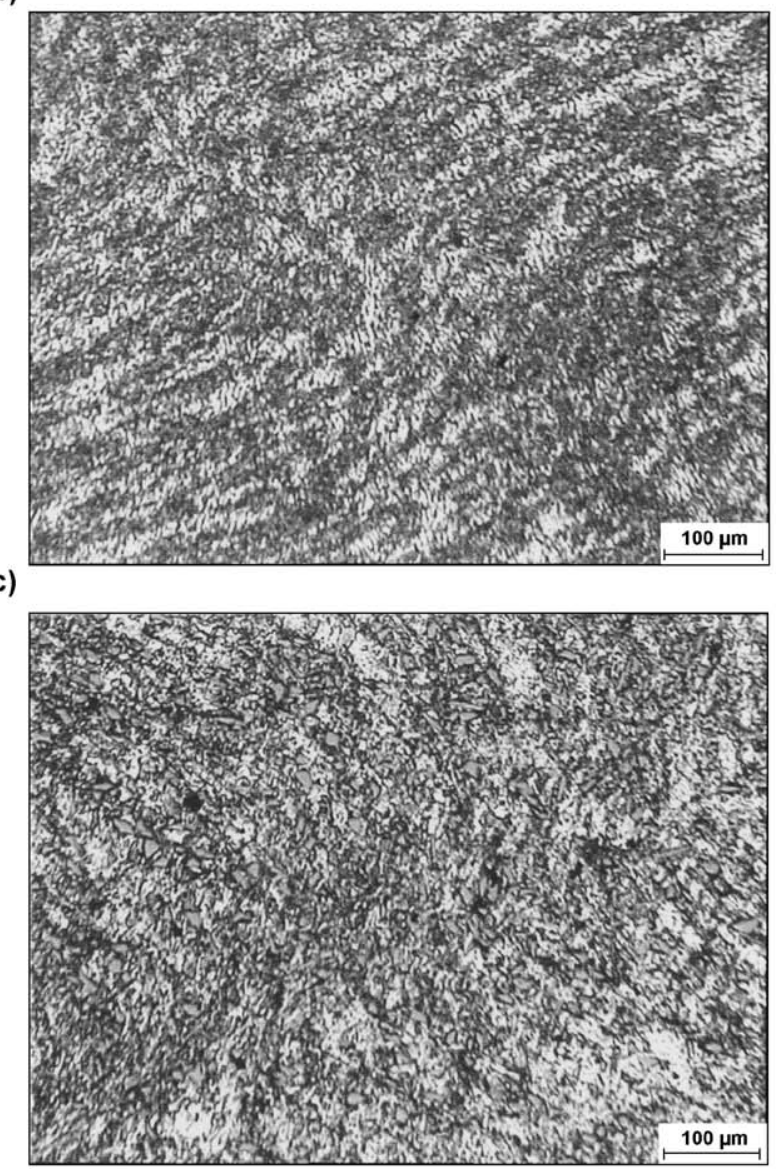

Fig 2. Deformation patterns of $\mathrm{SiCp} / 2024 \mathrm{Al}$ matrix composites: (a) particle diameter $3.5 \mu \mathrm{m}$, (b) particle diameter $10 \mu \mathrm{m}$, (c) particle diameter $20 \mu \mathrm{m}$.

by strain gradient strengthening effect. Furthermore, the smaller the particle size, the higher the strain gradient is introduced in the matrix and the better is the strengthening effect.

\subsection{Microscopic observations}

To examine the formation of adiabatic shear band in materials, microscopic observations for loaded specimens 
were carried out under NEOPHOT-21 light optical microscope. The typical micrographs for both $\mathrm{SiCp} / 2024 \mathrm{Al}$ composites are shown in Fig 2. From the micrographs, one can find that the formation of adiabatic shear banding depends strongly on the reinforcing particle size. In the composite containing $3.5 \mu \mathrm{m} \mathrm{SiC} \mathrm{particles,} \mathrm{the} \mathrm{marked}$ localization of shear band is clearly visible (Fig. 2a). However, under the same magnification, the microscopic deformations in the other two composites containing 10 and $20 \mu \mathrm{m} \mathrm{SiC} \mathrm{particles} \mathrm{do} \mathrm{not} \mathrm{show} \mathrm{clear} \mathrm{localized} \mathrm{shear}$ band (Fig. $2 b$ and c). For example, in the composite containing $10 \mu \mathrm{m}$ of $\mathrm{SiC}$ particles, localization seemed to form but not evident, while in the composite containing 20 $\mu \mathrm{m}$ of $\mathrm{SiC}$ particles, no marked localized plastic flow pattern can be found. These experimental observations demonstrate that adiabatic shear banding can be more readily observed in small-particle composite.

\section{Analysis and discussions}

To study this size dependency adiabatic shear banding phenomenon, a strain gradient-dependent constitutive equation for MMCp was recently developed by the authors of the present paper [16]. For the present adiabatic shear banding problem, this strain gradient-dependent constitutive equation for MMCp can be written as:

$\tau=A \gamma^{n} \dot{\gamma}^{m} \theta^{-\lambda}(1+\hat{l} \eta)^{v}$

where $\gamma, \dot{\gamma}$, and $\theta$ are the shear strain, the shear strain rate, and the temperature, respectively, $A, n, m, \lambda$, and $v$ are positive empirical constants, $\tau$ is the shear flow stress of MMCp, $v$ is the positive dimensionless constant, $\hat{l}$ is the material length, and $\eta$ is the effective shear strain gradient and its explicit expression can be found in our recent paper [16]. According to the instability condition for the maximal shear stress $\mathrm{d} \tau / \mathrm{d} \gamma=0$, the explicit expression for the critical instability strain $\gamma_{c}$ is given by

$\gamma_{\mathrm{c}}=\left[\frac{n \rho C}{K A \lambda \dot{\gamma}^{m}(1+\hat{l} \eta)^{v}}\right]^{1 / \eta+1} \theta^{(\lambda+1) /(\eta+1)}$

where $\rho$ and $C$ are the density and the specific heat, respectively, and $K$ is the so-called Taylor-Quinney coefficient, usually, $K \cong 0.9$. It is clearly seen from Eq. (2) that the critical strain $\gamma_{\mathrm{c}}$ decreases as the strain gradient $\eta$ increases. This means that high strain gradient provides a strong driving force for the formation of adiabatic shear band.

From the aforementioned experimental observations, one can find that the localized shear banding is more clearly observed in the composite reinforced with the small particles than that in the composite with larger particles. Actually, for a MMCp, the reinforcing particles deform elastically, and the plastic deformation only occurs in the metal matrix. Obviously, the spacing between particles is an important geometrical parameter for controlling an inhomogeneous plastic deformation in the composite [17]. According to our previous study $[15,16]$, this inhomogeneous plastic deformation can be effectively characterized by the strain gradient. Furthermore, the smaller the particle size is, the smaller the particle spacing is and the higher the strain gradient is in the composite if the volume fraction of the particle is kept fixed. In a microscopic view, high strain gradient will promote the development of the localized plastic deformation. On the other hand, the composite with the smallest particles is of the highest flow stress and its dissipated plastic work is greater than other large particle cases. This, in turn, causes a rapid increase in temperature and produces thermal softening, promoting the onset of adiabatic shear banding. The current simple instability analysis is in good accordance with the aforementioned experimental observations.

\section{Conclusions}

In this paper, the effect of the particle size on the formation of adiabatic shear banding in MMCp was investigated by making use of split Hopkinson pressure bar (SHPB). The experimental results demonstrate that the formation of adiabatic shear banding depends strongly on the reinforcing particle size, namely, adiabatic shear banding is more readily found in the composite with the small particles than that in the composites with larger particles. Further analysis shows that high strain provides a strong driving force for the formation of adiabatic shear banding in MMCp.

\section{Acknowledgements}

The authors gratefully acknowledge the financial support of this work by the National Natural Science Fund of China (No. 10232040).

\section{References}

[1] C. Zener, J.H. Hollomon, J. Appl. Phys. 14 (1944) 22.

[2] H.C. Rogers, Int. Rev. Mater. Sci 9 (1979) 283.

[3] R.J. Clifton, Material response to ultra high loading rates. NRC Report No. 356, Washington (DC): US National Material Advisor Board, 1980, Chapter 8.

[4] Y.L. Bai, J. Mech. Phys. Solids 30 (1982) 195.

[5] A. Molinari, R.J. Clifton, J. Appl. Mech. 54 (1987) 806.

[6] Y.L. Bai, B. Dodd, Adiabatic Shear Localization, Pergamon, Oxford, 1992. Chapter 2.

[7] K.T. Ramesh, Mech. Mater. 17 (1994) 165.

[8] M.A. Meyers, Dynamic Behavior of Materials, Wiley, New York, 1994. Chapter 15.

[9] M.A. Meyers, V.F. Nesterenko, J.C. LaSalvia, Q. Xue, Mater. Sci. Eng. A317 (2001) 204. 
[10] E. El-Magad, J. Phys. III C3 (1997) 511.

[11] S. Lee, K.M. Cho, K.C. Kim, W.B. Choi, Metall. Trans. 24A (1993) 895.

[12] Z. Ling, L. Luo, B. Dodd, J. Phys. IV 4 (1994) 453.

[13] D.R. Chichili, K.T. Ramesh, Int. J. Solids Struct. 17/18 (1995) 2609.
[14] L.H. Dai, Y.L. Bai, J. Compos. Mater. 32 (1998) 246.

[15] L.H. Dai, Z. Ling, Y.L. Bai, Compos. Sci. Technol. 61 (2001) 1057. [16] L.F. Liu, L.H. Dai, G.W. Yang, Mater. Sci. Eng. A345 (2003) 190.

[17] S.V. Kamat, A.D. Rollett, J.P. Hirth, Scr. Metall. Mater. 25 (1991) 27. 